\title{
Vacuna conjugada polisacárida contra la neumonía neumocócica en adultos
}

\author{
Polysaccharide conjugate vaccine against pneumococcal pneumonia in adults
}

\section{Objetivos}

Evaluar la efectividad de la vacuna neumocócica conjugada de 13 serotipos (VCN13) en adultos mayores de 65 años.

\section{Diseño, lugar y participantes}

Estudio aleatorizado, doble ciego, controlado con placebo, llevado a cabo en los Países Bajos entre septiembre de 2008 y agosto de 2013. Se incluyeron 84.496 pacientes adultos mayores de 65 años, 42.240 recibieron VCN13 y 42.256 recibieron placebo.

\section{Medición de resultados principales}

El resultado principal fue el primer episodio de neumonía adquirida de la comunidad (NAC) definida por la presencia de dos o más criterios clínicos pre-especificados, sumado a hallazgos compatibles en una radiografía de tórax, y la detección de antígenos urinarios, o aislamiento de un serotipo de S. pneumonie cubierto por la vacuna, en sangre u otro tejido estéril. Los resultados secundarios fueron NAC no bacteriémica y no invasiva, o enfermedad neumocócica invasiva (ENI). El análisis se realizó prioritariamente por protocolo (pacientes que tuvieron un episodio de NAC o ENI luego de 14 días de la fecha de vacunación, recibieron vacuna o placebo y no presentaron desviaciones del protocolo).

\section{Resultados}

Luego de una media de seguimiento de 3,97 años, se observaron los resultados que se muestran en la tabla 1. VCN13 fue eficaz en la prevención de NAC y ENI en adultos mayores de 65 años con persistencia de la eficacia durante al menos cuatro años. En el análisis modificado por intención de tratar, se observó una eficacia similar (eficacia de la vacuna para NAC, neumonía no bacteriémica no invasiva y ENI de $37,7 \%, 41,1 \%$ y $75,8 \%$, respectivamente). El número de eventos adversos graves y muertes fue similar en los dos grupos, pero hubo más reacciones locales en el grupo VCN13. Este ensayo no identificó problemas de seguridad asociados con el uso de VCN13 en los más de 42.000 pacientes que fueron vacunados.

Tabla 1. Eficacia de la vacuna en la primo-infección por serotipos presentes en la vacuna y neumonía adquirida en la comunidad de cualquier causa.

\begin{tabular}{|c|c|c|c|c|}
\hline \multirow[t]{2}{*}{ Resultado } & $\begin{array}{c}\text { VCN } 13 \\
(\mathrm{~N}=42.240)\end{array}$ & $\begin{array}{c}\begin{array}{c}\text { Placebo } \\
\text { (N=42.256) }\end{array}\end{array}$ & \multirow[t]{2}{*}{$\begin{array}{l}\text { Eficacia vacuna* \% } \\
\text { (IC 95\%) }\end{array}$} & \multirow[t]{2}{*}{ p } \\
\hline & \multicolumn{2}{|c|}{ Episodios } & & \\
\hline Neumonía adquirida de la comunidad confirmada & 49 & 90 & $45,6(21,8 \text { a } 62,5)^{a}$ & $<0,001$ \\
\hline Neumonía no bacteriémica y no invasiva confirmada & 33 & 60 & $45,0(14,2 \text { a } 65,3)^{a}$ & 0,007 \\
\hline Enfermedad invasiva por neumococo & 7 & 28 & $75,0(41,4 \text { a } 90,8)^{a}$ & $<0,001$ \\
\hline Neumonía adquirida en la comunidad de cualquier causa & 747 & 787 & $5,1(-5,1 \text { a } 14,2)^{\beta}$ & 0,32 \\
\hline
\end{tabular}

VCN 13: vacuna neumocóccica conjugada de 13 serotipos. IC95\%: Intervalo de confianza. $\square$ Análisis por protocolo. $\square$ Análisis por intención de tratar modificado. * La eficacia de una vacuna se define como el porcentaje de reducción de enfermedad en el grupo de individuos vacunados comparado con el grupo no vacunado, utilizando las condiciones más favorables.

\section{Conclusiones}

En los adultos mayores VCN13 fue efectiva para prevenir la NAC, neumonía no bacteriémica y no invasiva y ENI provocados por serotipos cubiertos por la vacuna, pero no en prevenir la NAC de cualquier causa.
Fuente de financiamiento: estudio colaborativo entre el Centro Médico de la Universidad Utrecht y Wyeth (una empresa de Laboratorios Pfizer) como patrocinante.

\section{Comentario}

Las vacunas antineumocócicas conjugadas, primero la de siete serotipos (VCN7) y luego la VCN13 se utilizan en menores de dos años desde el año 2000. Desde 2012, y en base a estudios de inmunogenicidad de VCN13 en adultos, de eficacia de VCN7 en pacientes con VIH/SIDA, y teniendo en cuenta la alta carga de morbimortalidad en los grupos de mayor riesgo de ENI, diferentes organizaciones nacionales e internacionales han recomendado la vacunación secuencial con VCN13 y vacuna polisacárida de 23 serotipos (VPN23) en poblaciones adultas con factores de riesgo $^{1-3}$. La decisión de recomendar VCN13 entre los adultos sanos mayores de 65 años llegó de la mano del estudio que se comenta aquí, y a partir de su publicación las diferentes entidades han recomendado el agregado de VCN13 a los esquemas de vacunación antineumocócica existentes ${ }^{4-5}$. El objetivo de este esquema secuencial es proveer mayor inmunogenicidad con VCN13 y al mismo tiempo mayor espectro de protección de serotipos con la VPN23.

Conclusiones del comentador

Actualmente el esquema de vacunación para pacientes sanos mayores de 65 años consiste en una dosis de VCN13 seguida de una dosis de VPN23 con un intervalo de 1 año. En caso de haber recibido una dosis de VPN23 inicial, se recomienda administrar una dosis de VCN13 con un intervalo de por lo menos un año ${ }^{5}$.

Corina Nemirovsky [Sección Infectología, Servicio de Clínica Médica del Hospital Italiano de Buenos Aires.

corina.nemirovsky@ hospitalitaliano.org.ar]

Nemirovsky C. Vacuna conjugada polisacárida contra la neumonía neumocócica en adultos. Evid. Act Pract Ambul. 2017;20(1):4. Comentario de: Bonten MJM, y col. Polysaccharide Conjugate Vaccine against Pneumociccal Pneumonia in Adults: a randomided controlled trial. N Engl J Med 2015;372:1114-25. PMID: 25785969.

\section{Referencias}

1. Moberley et al. Vaccines for preventing pneumococcal infections in adults. Cochrane Databe Syst Rev 2008; CD000422

2. Jackson LA, y col. Immunogenicity and safety of a 13-valent pneumococcal conjugate vaccine in adults 70 years of age and older previously vaccinated with 23 -valent pneumococcal polysaccharide vaccine. Vaccine 2013;31(35):3585-3593.

3. Goldblatt D, y col. The Immunogenicity of 7-Valent Pneumococcal Conjugate Vaccine versus 23-Valent Polysaccharide Vaccine in Adults Aged 50-80 Years. Clinical Infectious Diseases 2009;49(9):1318-1325

4. Conjugate Vaccine and 23-Valent Pneumococcal Polysaccharide Vaccine Among Adults Aged > 65 Years: Recommendations of the Advisory Committee on Immunization Practices (ACIP). MMWR 2014;63:822-825.

5. Nemirovsky C. Puesta al día en vacunación antineumocócica en adultos. Rev. Hosp. Ital. B.Aires 2015; 35(3): 97-101. 